\title{
Liouville-von Neumann approach and time-dependent Gaussian approximation
}

\author{
Hyeong-Chan $\mathrm{Kim}^{\dagger}$ 円 and Jae Hyung Yee ${ }^{\dagger \star}$ 円 \\ ${ }^{\dagger}$ Institute of Basic Science, Yonsei University, Seoul 120-749, Korea \\ * Institute of Physics and Applied Physics, Yonsei University, Seoul 120-749, Korea
}

(October 22, 2018)

\begin{abstract}
We show that Liouville-von Neumann approach to quantum mechanical systems, which demands the existence of invariant operators, reproduces the timedependent variational Gaussian approximation. We find the effective action of the time-dependent systems and show that many aspects of the dynamics are independent of the details of time evolution, e.g., the squeezing of the wave-function is determined by the effective potential of the final stage of time-evolution.
\end{abstract}

Keywords: Liouville-von Neumann approach, Gaussian, slow-rolling

PACS numbers: 05.70.Ln, 11.80.Fv, 98.80.Cq

\footnotetext{
${ }^{1}$ E-mail: hckim@phya.yonsei.ac.kr

${ }^{2}$ E-mail: jhyee@phya.yonsei.ac.kr
} 


\section{Introduction}

The interests in searching for a method which effectively describes the real-time quantum dynamics has been continually increased, in relation with the quantum theory out of thermal equilibrium [四], the inflationary scenario of the Universe [2], the dynamic process in phase transitions [3], the formation of topological defects in variety of phenomena in condensed matter physics [4]. Understanding quantum mechanical evolution of inflaton field in the new inflationary universe is vital for correct determination of the inflationary epoch's period [5].

A time-dependent variational approximation for quantum dynamics was developed in Schrödinger picture [6, 7]. The Liouville-von Neumann (LvN) approach of the timedependent harmonic [8] and driven harmonic [9] oscillators have been developed to obtain the exact time-dependence of quantum states, and the methods are generalized to the cases of anharmonic oscillator, scalar inflaton field dynamics [10], and driven anharmonic oscillator 11]. LvN approach has a merit that it can be directly applied to both timeindependent and time-dependent quantum systems without any modification, because it is based on the operator level equations.

The purpose of this paper is to extend the approach developed in Ref. [10] to include arbitrary potential, so that one can develop a general Gaussian approximation using the LvN method. This approach has two merits: 1) it can describe the time-evolution of systems from the start to the end. 2) In LvN approach, it is easy to define the general Fock space because it directly identifies the creation and annihilation operators.

The organization of this paper is as follows. In Sec. II, the LvN approach is elaborated to be applicable to the case of general potential $V(\hat{q}, t)$ in obtaining the Gaussian approximation, and is applied to find the Fock space and the time-dependent expectation values of some operators for $V(\hat{q}, t)$. Sec. III is devoted to the study of a model with slow rolling potential. In Sec. IV, we summarize our results and present some discussions.

\section{Gaussian approximation in Liouville-von Neumann approach}

The quantization of a time-dependent anharmonic oscillator using the LvN approach was studied in Ref. [10]. It was shown that their approach for symmetric potential is equivalent to the variational Gaussian approximation and the mean-field, Hartree-Fock method. In this section, we develop LvN method for a general potential and show that this approach exactly reproduces the Gaussian approximation of Ref. [7], without losing the merits of the LvN approach.

We start with the Hamiltonian:

$$
\hat{H}(t)=\frac{\hat{p}^{2}(t)}{2 m(t)}+V(\hat{q}(t), t),
$$


where the potential is an arbitrary function of $\hat{q}(t)$ and $t$.

Now let us assume the time-evolution of an initial ground state can be described by a Gaussian wave-packet $\Psi(q, t)=r e^{-\frac{i v^{*}}{2 u^{*}} q^{2}-i \frac{x^{*}}{u^{*}} q}$, which is annihilated by an operator:

$$
\hat{A}=i\left[u^{*}(t) \hat{p}(t)+v^{*}(t) \hat{q}(t)+x^{*}\right] .
$$

The $\mathrm{LvN}$ approach is to demand this operator to be invariant under time evolution:

$$
0=\frac{d \hat{A}}{d t}=i\left[\dot{u}^{*}+\frac{v^{*}}{m(t)}\right] \hat{p}(t)+i\left[\dot{v}^{*} \hat{q}(t)-u^{*} V^{\prime}(\hat{q}, t)+\dot{x}^{*}\right] .
$$

Taking the expectation values with respect to the wave-function $\Psi$ on each side of the equations obtained from the variations in $\hat{p}$ or $\hat{q}$ of Eq. (3), gives

$$
\begin{aligned}
& v^{*}(t)=-m(t) \dot{u}(t), \quad \dot{v}(t)-u(t)\left\langle V^{\prime \prime}(\hat{q}, t)\right\rangle=0, \\
& \dot{x}^{*}(t)+u^{*}(t)\left[\langle\hat{q}(t)\rangle\left\langle V^{\prime \prime}(\hat{q}, t)\right\rangle-\left\langle V^{\prime}(\hat{q}, t)\right\rangle\right]=0 .
\end{aligned}
$$

These equations reduce to one equation for $u$ :

$$
m(t) \ddot{\bar{u}}+\left\langle V^{\prime \prime}(\hat{q}, t)\right\rangle \bar{u}(t)=0,
$$

where $\bar{u}(t)=\sqrt{m(t)} u(t)$. This equation is too complexly coupled to $x(t)$ to be solved exactly, except for a simple problem of harmonic oscillators [11]. To overcome this difficulty and to find a way for easier application to physical problems, we define an auxiliary function

$$
\bar{q}(t)=-i \hbar\left[\bar{u}(t) x^{*}(t)-\bar{u}^{*}(t) x(t)\right],
$$

which satisfies the equation of motion

$$
\ddot{\bar{q}}(t)+\frac{\left\langle V^{\prime}(\hat{q}, t)\right\rangle}{\sqrt{m(t)}}=0 .
$$

The equation (8) becomes the ordinary differential equation for $\bar{q}$ only in the semiclassical approximation. In general, the expectation value $\langle F(\hat{q}, t)\rangle$ depends on $\bar{q}$ and $r^{2}=\hbar|\bar{u}|^{2}$. Therefore, we need also to consider the equation of motion for $r$ [11]:

$$
\ddot{r}-\frac{L^{2}}{r^{3}}+\frac{\left\langle V^{\prime \prime}(\hat{q}, t)\right\rangle}{m(t)} r=0,
$$

where $L=r^{2}\left(t=t_{0}\right) \dot{\phi}\left(t=t_{0}\right)$ is a constant of motion, with $\phi$ being the phase part of $u(t)$. If the initial wave-packet at origin is a static Gaussian, the initial condition becomes $\bar{u}_{i}(t)=\frac{1}{\sqrt{2 \hbar \omega_{0}}} e^{-i \omega_{0} t}$ and $x(0)=0$, which gives $L=-1 / 2$. The two equations (8), (9) and the boundary condition mentioned above determine a unique solution for $\bar{q}$ and $r$. 
Once we get $u$ and $x$ from Eqs. (4), (5), (8), and (9), the time dependence of quantum dynamical operators is explicitly written as:

$$
\begin{aligned}
& \hat{q}(t)=u(t) \hat{A}+u^{*}(t) \hat{A}^{\dagger}+\frac{\bar{q}(t)}{\sqrt{m(t)}}, \\
& \hat{p}(t)=m(t)\left\{\dot{u}(t) \hat{A}+\dot{u}^{*}(t) \hat{A}^{\dagger}+\frac{d}{d t} \frac{\bar{q}(t)}{\sqrt{m(t)}}\right\} .
\end{aligned}
$$

Therefore, the expectation values of operators at time $t$ for the state $\Psi$ are given by

$$
\begin{aligned}
\langle\hat{q}(t)\rangle & =\frac{\bar{q}(t)}{\sqrt{m(t)}}, \quad\langle\hat{p}(t)\rangle=m(t) \frac{d}{d t} \frac{\bar{q}(t)}{\sqrt{m(t)}} \\
\left\langle\hat{q}^{2}(t)\right\rangle & =\left[\frac{\bar{q}(t)}{\sqrt{m(t)}}\right]^{2}+\hbar^{2}|u|^{2}, \quad\left\langle\hat{p}^{2}(t)\right\rangle=\left[m(t) \frac{d}{d t} \frac{\bar{q}(t)}{\sqrt{m(t)}}\right]^{2}+m^{2}(t) \hbar^{2}|\dot{u}|^{2}
\end{aligned}
$$

To have explicit form of Eqs. (4) and (8), we need to expand the expectation value of operator $F(\hat{q}, t)$ in series around $\bar{q}$ :

$$
\langle F(\hat{q}, t)\rangle=\sum_{n=0}^{\infty} \frac{F^{(n)}(\bar{q} / \sqrt{m})}{n !}\left\langle[\hat{q}(t)-\bar{q} / \sqrt{m(t)}]^{n}\right\rangle,
$$

where the expectation values of the powers of $[\hat{q}(t)-\bar{q} / \sqrt{m(t)}]$ can be obtained from the iteration formula:

$$
\left\langle[\hat{q}(t)-\bar{q} / \sqrt{m(t)}]^{n}\right\rangle=(n-1) \hbar^{2}|u(t)|^{2}\left\langle[\hat{q}(t)-\bar{q} / \sqrt{m(t)}]^{n-2}\right\rangle, \quad n \geq 2 .
$$

Note that this series is the same as the series expansion in $\hbar$ because $\hbar^{2}|u|^{2}$ is $O(\hbar)$.

The equations (5) and (6) become to $O(\hbar)$,

$$
\begin{aligned}
\dot{x}^{*}(t)+u^{*}(t)\left[\frac{\bar{q}}{\sqrt{m}} V^{(2)}-V^{(1)}+\frac{\hbar^{2}|\bar{u}|^{2}}{2 m}\left(\frac{\bar{q}}{\sqrt{m}} V^{(4)}-V^{(3)}\right)\right] & =0, \\
m(t) \ddot{\bar{u}}(t)+\left[V^{(2)}+\frac{\hbar^{2}|\bar{u}|^{2}}{2 m} V^{(4)}\right] \bar{u}(t) & =0
\end{aligned}
$$

where $V^{(n)}=\left.\partial_{x}^{n} V(x, t)\right|_{x=\bar{q} / \sqrt{m}}$ is independent of $r$. The induced set of equations (8) and (9) is given by

$$
\begin{aligned}
\ddot{r}-\frac{1}{4 r^{3}}+V^{(2)} r+\frac{\hbar r^{3}}{2} V^{(4)} & =0 \\
\ddot{\bar{q}}(t)+\frac{V^{(1)}}{\sqrt{m(t)}}+\frac{\hbar r^{2}}{2 \sqrt{m(t)}} V^{(3)} & =0 .
\end{aligned}
$$


These equations of motion admit to define the effective Hamiltonian,

$$
\begin{aligned}
H_{e f f}(\bar{q}, \bar{r}) & =\frac{\dot{\bar{q}}^{2}}{2}+\frac{\dot{\bar{r}}^{2}}{2}+V^{(0)}+\frac{\bar{r}^{2}}{2} V^{(2)}+\frac{\hbar^{2}}{8 \bar{r}^{2}}+\frac{\bar{r}^{4} V^{(4)}}{8} \\
& =\frac{\dot{\bar{q}}^{2}}{2}+\frac{\dot{\bar{r}}^{2}}{2}+\langle V(\hat{q}, t)\rangle+\frac{\hbar^{2}}{8 \bar{r}^{2}},
\end{aligned}
$$

where $\bar{r}=\sqrt{\hbar} r=\hbar|\bar{u}|$. Eq. (19), which is a classical Hamiltonian for an anharmonic oscillator in two dimensional space $x^{i}=(\bar{q}, \bar{r})$, represents the same effective potential of Cooper, Pi, and Stancioff [7]. In this sense, the present approach exactly reproduces the variational gaussian approximation of previous authors. The LvN method, however, presents better understanding of the quantum mechanical system, because not only it naturally defines the complete Fock space through $A$ and $A^{\dagger}$ but also these operators are time independent. In this sense, the parameterizations of the wave-function by $u$ and $x$ is more convenient than by $q$ and $r$.

From the vacuum defined by $A|0\rangle=0$, we can naturally define the excited states by successively applying the creation operator $A^{\dagger}$ on the ground state. The mixed states can also be defined. For example, the thermal states can be defined from the density matrix [10]

$$
\hat{\rho}_{T}(t)=\frac{1}{Z_{N}} e^{-\beta \hbar \omega_{0}[\hat{N}(t)+1 / 2]},
$$

where $N(t)=\hat{A}^{\dagger} \hat{A}, \beta$ is the inverse temperature, $\omega_{0}$ is the frequency of $u$ at $t=0$, and the partition function $Z_{N}$ is given by

$$
Z_{N}=\sum_{n=0}^{\infty}\left\langle n\left|e^{-\beta \hbar \omega_{0}[\hat{N}(t)+1 / 2]}\right| n\right\rangle=\frac{1}{2 \sinh \left(\frac{\beta \hbar \omega_{0}}{2}\right)} .
$$

We explicitly write down the expectation values of some operators with respect to the thermal state:

$$
\begin{aligned}
\langle\hat{q}(t)\rangle_{T} & =\bar{q}(t), \quad\left\langle\delta \hat{q}^{2}(t)\right\rangle_{T}=\hbar^{2}|u(t)|^{2} \operatorname{coth} \frac{\beta \hbar \omega_{0}}{2} \\
\langle\hat{p}(t)\rangle_{T} & =m \dot{\bar{q}}(t), \quad\left\langle\delta \hat{p}^{2}(t)\right\rangle_{T}=\hbar^{2} m^{2}|\dot{u}(t)|^{2} \operatorname{coth} \frac{\beta \hbar \omega_{0}}{2} .
\end{aligned}
$$

\section{Slow rollover transition}

We set $m(t)=1$ in this section, since the mass dependence can be removed by reparametrization of other parameters in the Hamiltonian. We thus consider the potential

$$
V(\hat{q}, t)=\frac{\lambda}{4 !} \hat{q}^{2}(t)\left\{[\hat{q}(t)-a(t)]^{2}+k(t)\right\}
$$


where we assume $a^{2}(t)[\geq-k(t)]$ increases from zero to some constant number $a^{2}$, and $k(t)$ decreases from some positive number to a fixed non-positive number $\kappa$ at $t \rightarrow \infty$, which assures the potential at $q=0$ to be locally stable false vacuum. The dynamics for this potential contains many interesting quantum mechanical features depending on the time-dependence of $k(t)$ and $a(t)$, such as tunnelling $\left(-a^{2}<\kappa<0\right)$, phase transition $(\kappa=0)$, or slow rollover $\left(\kappa=-a^{2}\right)$.

The energy for a static Gaussian wave-packet at $t=0$ described earlier is given by

$$
E=\frac{1}{4} \hbar \omega_{0}+\frac{\lambda}{48} \hbar\left(a^{2}+k\right)+\frac{\lambda \hbar^{2}}{32 \omega_{0}^{2}}
$$

The effective potential corresponding to Eq. (19) becomes

$$
V_{e f f}(\bar{q}, \bar{r})=V(\bar{q}, t)+\frac{\lambda \bar{r}^{2}}{4}\left[\bar{q}^{2}-a \bar{q}+\frac{a^{2}+k}{6}\right]+\frac{\hbar^{2}}{8 \bar{r}^{2}}+\frac{\lambda \bar{r}^{4}}{8} .
$$

In the case of a time-independent system, we can describe the whole dynamics in terms of this effective potential. We present a contour plot of the effective potential in Fig. 1.

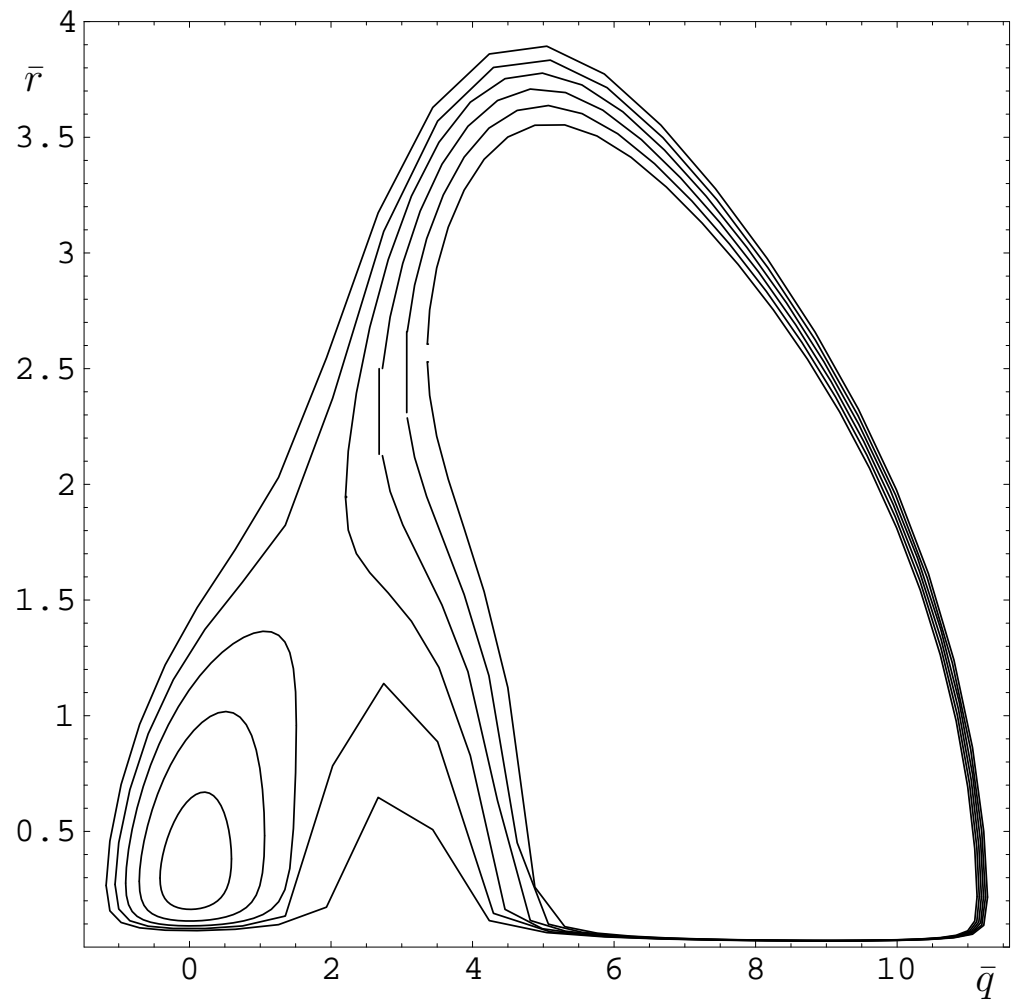

Fig. 1. The equipotential curves of the effective potential. The horizontal axis represents $\bar{q}$ and the vertical axis $\bar{r}$. In this figure we use the parameters, $a=8, k=-10, \hbar^{2} / \lambda=0.1$, and each curve represents the energy values, from the innermost one to outer direction, $\hbar^{2} E / \lambda=0,1,2,3,4,5$. A given Gaussian wave-function cannot escape the contour defined by its energy. 
One crucial difference of the present paper from that of Cooper et. al. is that we keep $\bar{r}$ dependence on the effective potential, while it was variationally determined in Ref. [6]. This results in a large difference in the slow rolling period. As clearly shown in Fig. 1, a given wave-packet has allowed region of $(\bar{q}, \bar{r})$ determined by the value of its energy. Depending on the initial value of $(\bar{q}, \bar{r})$, the wave-function simply oscillates around the false vacuum $\bar{q}=0$ or go over to the true vacuum near $\bar{q}=8$. There are two properties which are distinguished from its classical dynamics. First, there is a quantum mechanical driving force at $\bar{q}=0$. The classical stable equilibrium at $\bar{q}=0$ is not the equilibrium any more in quantum mechanics. Second, it was shown that as the dispersion (frequency) becomes smaller (larger), it becomes easier for $\bar{q}$ to go over the classical potential barrier [6]. In Fig. 1, it is evident that as the dispersion (frequency) becomes larger (smaller), it also becomes easier for $\bar{q}$ to go over the classical potential barrier. There is an inevitable restriction of the Gaussian approximation. In the presence of leaking into the right well the wave-function cannot be described by the Gaussian. Due to this restriction, we cannot effectively describe tunnelling process. To remove this restriction, one needs double Gaussian (Appendix B of Ref. [0]) or other approximations, which is out of scope of the present paper. If $a(t)$ becomes timedependent, the dynamics cannot be understood simply by the effective potential, and the equations of motion should be considered explicitly.

As an explicit dynamical example, we consider the slow rolling $\left(k+a^{2}=0\right.$ for $\left.t>0\right)$ potential. The equipotential curves are shown in Fig. 2. An interesting features of this effective potential is that the unstable equilibrium point at $\bar{q}=0$ disappears.

To understand the dynamics of this system, we write down the equation of motion for $x^{i}=(\bar{q}, \bar{r})$ explicitly:

$$
\ddot{x}^{i}+\nabla_{i} V_{e f f}\left(x^{j}\right)=0
$$

where $V_{\text {eff }}\left(x^{j}\right)$ is given by Eq. (25). Let a Gaussian wave-function prepared at $\bar{q}=0$ with velocity $\dot{q}(0)=0$ starts to evolve. In the case of slow rollover, the evolution is divided into three phases; the slow-rolling, rolling, and oscillating phases.

As soon as the packet released, it starts to roll down the potential wall. In the region $\bar{q} \sim 0, \bar{r}$ increases slowly until $\bar{r} \sim\left[\hbar^{2} /(2 \lambda)\right]^{1 / 6}$, which is the variational minimum of the potential in $\bar{r}$ at $\bar{q}=0$, because $\ddot{\bar{r}} \sim \frac{\sqrt{\hbar}}{4}\left(2 \omega_{0}\right)^{3 / 2}$ and $\dot{\bar{r}}(0)=0$. This time $\left(t_{1}\right)$, which $\bar{r}(t)$ takes in reaching to its variational value, is roughly given by $t_{1} \sim \frac{1}{(2 \lambda \hbar)^{1 / 12}}\left(\frac{2}{\omega_{0}}\right)^{3 / 4}$. After that, the time evolution of $(\bar{q}, \bar{r})$ is similar to its variational path. The initial time evolution of $\bar{q}$ is governed by the quantum-mechanical driving force given by $a(t)$ until $\bar{q}^{2} \simeq \bar{r}^{2}$. Therefore the time $\left(t_{2}\right)$ for $\bar{q}$ to increase to $\bar{r}$, can be obtained from

$$
\bar{q}\left(t_{2}\right) \simeq \frac{\lambda}{4} \int^{t_{2}} d t^{\prime} \int^{t^{\prime}} d t^{\prime \prime} \bar{r}^{2}\left(t^{\prime \prime}\right) a\left(t^{\prime \prime}\right) \sim \bar{r}\left(t_{2}\right) \sim \bar{r}(\bar{q})
$$




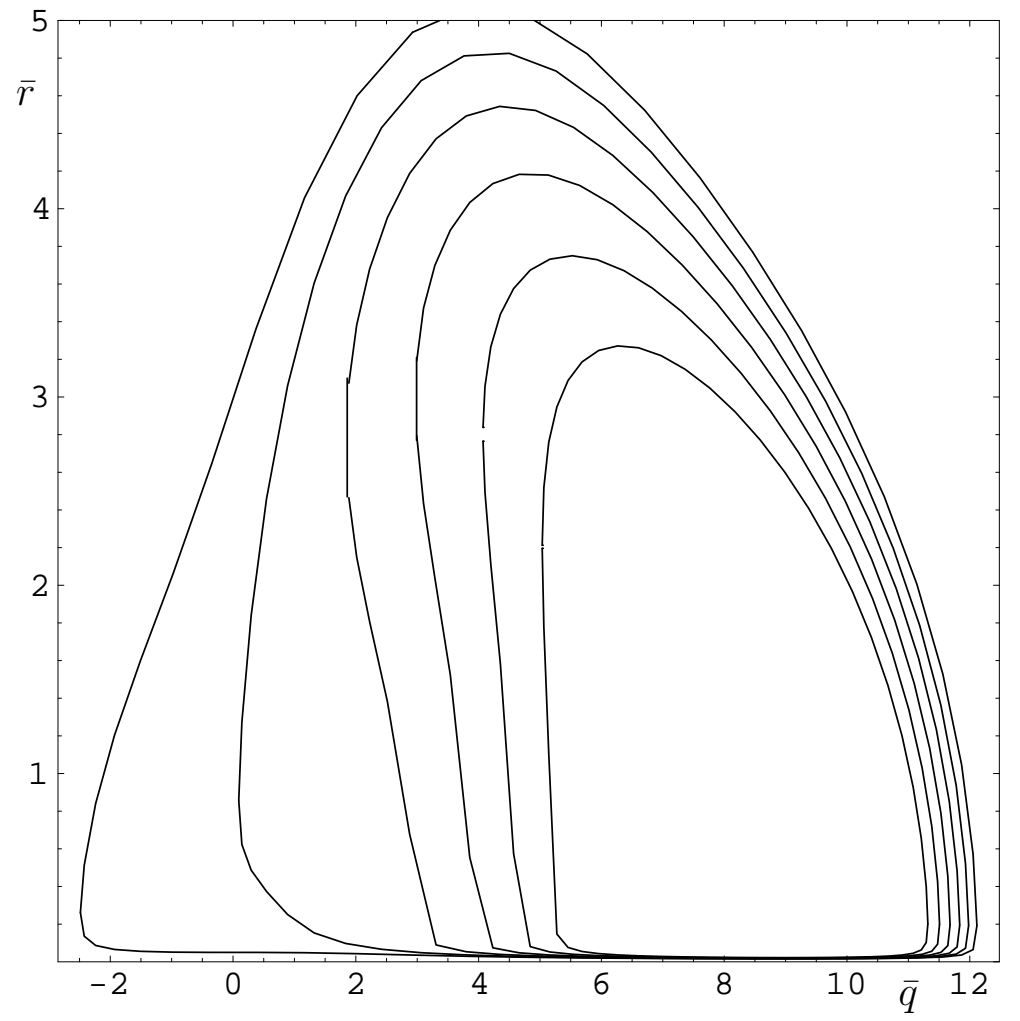

Fig. 2. The equipotential lines of a slow rolling transition. The horizontal axis represents $\bar{q}$ and the vertical axis $\bar{r}$. In this figure, $a=6, \hbar^{2} / \lambda=0.1$, and each curve represents the energy values $\hbar^{2} E / \lambda=10,0,-10,-20,-30,-40$ from outside. A given Gaussian wave-function cannot escape the contour defined by its energy.

If $t_{1}>t_{2}$, we determine $t_{2}$ from Eq. (27), which gives $t_{2} \sim\left(\frac{8 \cdot 5 !^{2}}{\lambda^{2} a^{2} \omega_{0}^{3} \hbar}\right)^{1 / 8}$. Otherwise, $t_{2}$ is given by the sum of the time taken by $\bar{r}(t)$ to increase to the variational value, $t_{1}$, and the variational time for the remaining part of $\bar{q}, t_{v}$, which will be shown shortly. The variational effective potential is $V_{v a r}=V_{\text {eff }}(\bar{q}, \bar{r}(\bar{q}))$, where $\bar{r}(\bar{q})$ satisfies

$$
\bar{r}^{6}(\bar{q})+\left(\bar{q}^{2}-a \bar{q}^{2}\right) \bar{r}^{4}(\bar{q})-\frac{\hbar^{2}}{2 \lambda}=0
$$

Therefore, in the variational approach, $\ddot{\bar{q}}(0)=\frac{\lambda a}{4}\left(\frac{\hbar^{2}}{2 \lambda}\right)^{1 / 3}$, and the duration of slowrolling phase is $t_{v} \sim \sqrt{\frac{8}{\lambda a}}$. Note that the duration $\left(t_{s}=t_{1}\right.$ or $\left.t_{s}=t_{2}+t_{v}\right)$ of the slowrolling phase in both of the scenario is considerably larger than the time determined by the variational method $t_{v}$.

For $t>t_{s}$, the wave-packet runs into the rolling phase. In this phase, the equation of motion becomes too difficult to get exact answer without numerical analysis. In this paper we simply outline the evolution without giving explicit solution. If $a(t)=a$ is a 
constant for $t>t_{s}$, and $\bar{r}^{2}$ is smaller than $\bar{q}^{2}$, one gets $q(t)$ from the elliptic integral

$$
\int^{\bar{q}} \frac{d q}{\sqrt{c+q^{2}\left(-2 a q+q^{2}\right)}}=\frac{\lambda}{12}\left(t-t_{s}\right)
$$

where $c=12 \dot{\bar{q}}^{2}\left(t_{s}\right) / \lambda$. However, as one can see from Eq. (26), $\bar{r}$ grows quickly during the period $\bar{r}^{2}<a \bar{q}-\bar{q}^{2}$, which lasts until $\bar{q} \simeq a$. Once $\bar{r}$ or $\bar{q}$ grows enough and fails to satisfies the condition, $\bar{r}^{2}<a \bar{q}-\bar{q}^{2}, \bar{r}$ goes into the oscillating phase.

After $\bar{q}$ reaches to the potential minima, it starts to oscillate around the minimum of the potential, which is the last stage. We do not restrict to the slow rolling case in the calculation of this stage. We simply assume that the final wave-packet resides around the true vacuum, which is determined by $\left.\nabla V_{e f f}\right|_{\vec{x}_{0}}=0$. For a slow rolling potential, there is a unique point which satisfies this condition. Now, we series expand the potential around the true vacuum:

$$
V_{T}(\vec{x})=V_{e f f}\left(\vec{x}_{0}\right)+\left.\frac{1}{2} \sum_{i, j} \partial_{i} \partial_{j} V_{e f f}(\vec{x})\right|_{x=x_{0}}\left(x^{i}-x_{0}^{i}\right)\left(x^{j}-x_{0}^{j}\right)+\cdots
$$

The solutions for the equation of motion for this $V_{T}$ are given by two frequency series expansion in general:

$$
x^{i}(t)=x_{0}^{i}\left[1+\sum_{n=1}^{\infty} \sum_{m= \pm} \chi_{n, m} \cos n\left(\omega_{m} t+\theta_{m}\right)\right],
$$

where $\chi_{n, m}$ is determined from the differential equation (26) up to its scale, and $\omega_{ \pm}$is the normal mode frequencies of $V_{T}$. We show this fact to the first order in the series expansion. Using the coordinates transformation:

$$
X_{+}=\frac{\bar{q}+b \bar{r}}{\sqrt{1+b^{2}}}, \quad X_{-}=\frac{b \bar{q}-\bar{r}}{\sqrt{1+b^{2}}},
$$

the Hamiltonian, up to quadratic order, becomes

$$
H_{T}=\dot{X}_{+}^{2}+\dot{X}_{-}^{2}+\frac{1}{2}\left[\omega_{+}^{2} X_{+}^{2}+\omega_{-}^{2} X_{-}^{2}\right]
$$

where $b$ and $\omega_{ \pm}$satisfy

$$
\begin{aligned}
& b^{2}-\frac{\left.\partial_{q}^{2} V_{e f f}(\vec{x})\right|_{x=x_{0}}-\left.\partial_{r}^{2} V_{e f f}(\vec{x})\right|_{x=x_{0}}}{\left.2 \partial_{1} \partial_{2} V_{e f f}(\vec{x})\right|_{x=x_{0}}} b-1=0, \\
& \omega_{ \pm}^{2}=\frac{\left.\sum_{i} \partial_{i}^{2} V_{e f f}(\vec{x})\right|_{x=x_{0}}}{2}-\left.\frac{1+b^{2}}{b} \partial_{1} \partial_{2} V_{e f f}(\vec{x})\right|_{x=x_{0}} .
\end{aligned}
$$

The solutions for the Hamiltonian system (33) are $X_{ \pm}(t)=X_{0, \pm} \cos \left(\omega_{ \pm} t+\theta_{ \pm}\right)$, where $X_{0, \pm}$ should be determined from the whole time evolution. From this we get, to first order in $X_{0}$,

$$
\bar{q}(t)=q_{0}+\frac{X_{+}(t)+b X_{-}(t)}{\sqrt{1+b^{2}}}, \quad \bar{r}(t)=r_{0}+\frac{b X_{+}(t)-X_{-}(t)}{\sqrt{1+b^{2}}} .
$$


We have two unknown parameters $X_{0, \pm}$ which should be determined from the whole timeevolution. If the system is time independent for $t>0$, one of them can be determined from the energy conservation law.

We now turn our attention to the calculation of $\bar{u}(t)=r e^{-i \phi} / \sqrt{\hbar}$. This can be done by integrating $\dot{\phi}=1 /\left(2 r^{2}\right)$. But, this integration is highly nontrivial if $X_{0}$ is not much smaller than $r_{0}$, and the slow rolling is the case. As a round about method, we directly try to solve the equation for $u$, and then determine the free parameters in $u$ from the effective potential. To simplify the procedures, we assume $b \ll 1$ and $r_{0} \ll q_{0}$. These two assumptions are usually satisfied for large $\bar{q}_{0}$. Now we write

$$
\bar{q}=q_{0}[1-\chi \cos (\omega t+\theta)],
$$

where $q_{0} \chi=-X_{0,+}$. The equation (16) for $u$, to linear order in $\chi$, can be approximated to be:

$$
\ddot{\bar{u}}(t)+\omega^{2}\left[1-\chi^{\prime} \cos (\omega t+\theta)\right] \bar{u}(t)=0,
$$

where $\chi^{\prime}=\frac{\lambda\left(q_{0}^{2}-a q_{0} / 2\right)}{\omega^{2}} \chi$. With the transformation $\omega t+\theta=2 \phi$, Eq. (38) becomes the Mathieu equation with $s=2, \quad b=4+4 \chi^{\prime}$, and $h^{2}=8 \chi^{\prime}$ in page 562 of Ref. [12]. Its solution, to the first order in $\chi^{\prime}$, is given by

$$
\bar{u}(t)=\alpha u_{+}(t)+\beta^{*} u_{+}^{*}(t),
$$

where $\alpha$ and $\beta$ are constants, and $u_{+}(t)=\frac{1}{\sqrt{2 \hbar \omega}}\left[e^{-i(\omega t+\theta)}-\frac{\chi^{\prime}}{6} e^{-2 i(\omega t+\theta)}+\frac{\chi^{\prime}}{2}\right]$. Note that $u_{+}$satisfies $u_{+} \dot{u}_{+}^{*}-u_{+}^{*} \dot{u}_{+}=i$. Note also that

$$
\bar{r}^{2}(t)=\hbar^{2}|u(t)|^{2}=\hbar^{2}\left[\left(|\alpha|^{2}+|\beta|^{2}\right)\left|u_{+}(t)\right|^{2}+\alpha \beta u_{+}^{2}+\alpha^{*} \beta^{*} u_{+}^{* 2}\right],
$$

where its time average $\hbar\left(|\alpha|^{2}+|\beta|^{2}\right) /(2 \omega)$ should give $\bar{r}_{0}^{2}$. From this relation and Eq. (42) below, we determine $|\beta|=\sqrt{\omega r_{0}^{2} / \hbar-1 / 2}$. Yet undetermined parameters are $\chi$, the size of fluctuation of $\bar{q}$, and the relative phase between $\alpha$ and $\beta$, whose determinations need the analysis of whole time evolution.

After integrating the equation (5) in $t$ one obtains

$$
x(t)=x_{0}+q_{0}\left[\alpha \dot{u}_{+}(t)+\beta^{*} \dot{u}_{+}^{*}(t)\right] .
$$

By equating $-i \hbar\left[u(t) x^{*}(t)-u^{*}(t) x(t)\right]$ computed by (39) and (41) to $\bar{q}(t)$ in (37), one obtains the following two equations:

$$
\begin{aligned}
|\alpha|^{2}-|\beta|^{2} & =1, \\
x_{0} & =-i \sqrt{\frac{\omega}{2 \hbar}} q_{0} \chi\left(\alpha-\beta^{*}\right),
\end{aligned}
$$


where $x_{0}$ denotes the constant shifting of annihilation operator, which gives a constant coherence, and $\beta$ denotes the squeezing with respect to the true vacuum.

The invariant annihilation operator is related to the annihilation and creation operators at the true vacuum by the following relation:

$$
\begin{aligned}
\hat{A} & =i\left[\bar{u}^{*} \hat{p}-\dot{\bar{u}}^{*} \hat{q}+x^{*}\right] \\
& =\alpha^{*} \hat{a}+\beta \hat{a}^{\dagger}
\end{aligned}
$$

where $\hat{a}=i\left[u_{+}^{*}(t) \hat{p}(t)-\dot{u}_{+}^{*}(t)\left(\hat{q}(t)-q_{0}\right)+\sqrt{\frac{\omega}{2 \hbar}} q_{0} \chi\right]$ is the annihilation operator at $\bar{q}=$ $q_{0}$ with $O\left(\chi^{1}\right)$ constant coherence. We cannot determine all the parameters without solving the full time evolution of the system, which needs numerical work. However, many important parts of the dynamics are determined without explicitly solving the full dynamics, e.g., the squeezing and coherence are determined just by the effective potential at $t \rightarrow \infty$, which is new results.

\section{Summary and discussions}

We have developed a LvN approach which gives the time-dependent Gaussian approximation. We, then, applied the method to a system with time-dependent slow rolling type potential, and found the time evolution of a Gaussian wave-packet initially centered at $q=0$. We divided the evolution into three stages, and described solutions for each stages. We have calculated the period of the slow rolling phase $\left(t_{s}\right)$, which is significantly

larger compared to the variationally determined value $t_{v}=\sqrt{8 /(\lambda a)}$. We also displayed that many information of the final stage can be obtained without explicit solution of the full time evolution. Explicitly, we have shown that the invariant annihilation operator can be expressed as a linear combination of the creation and annihilation operators of true vacuum. We have also shown that the squeezing and constant coherence are determined only by the dynamics around the absolute minimum of the effective potential, independent of the details of the whole dynamics.

This approach is the synthesis of the merits of the Gaussian approximation and LvN method. In the point of view of Gaussian approximation, it shows exactly the same potential in $\bar{q}$ and $\bar{r}$ as that of Ref. [7], and in the point of view of LvN method, it was shown that the method can be applicable for general type of potential as a well posed approximation. There is, however, restrictions for the application range of the type of the potential. The Gaussian approach is not appropriate to describe the symmetry breaking

potential from the start to the end, even though it is fairly accurate until $\bar{r} \simeq \sqrt{\frac{2}{3}} a$. This limitation shown in Ref. [7] was not cured in this paper. To overcome this limitation we need double Gaussian trial wave function or other approximations.

\section{Acknowledgments}


This work was supported in part by Korea Research Foundation under Project number KRF-2001-005-D00010 (H.-C.K. and J.H.Y.). 


\section{References}

[1] O. Eboli, R. Jackiw, and So-Young Pi, Phys. Rev. D 37, 3557 (1988).

[2] A. H. Guth, Phys. Rev D 23, 347 (1981); A. D. Linde, Phys. Lett. B 114, 431 (1982).

[3] W. H. Zurek, Nature (London) 317, 505 (1985); Acta Phys. Pol. B 24, 1301 (1993).

[4] A. J. Bray, Adv. Phys. 43, 357 (1994).

[5] A. Guth and S.-Y. Pi, Phys. Rev. D 32, 1899 (1985).

[6] F. Cooper and So-Young Pi, "Time-dependent variational principle for quantum dynamics in the Schrodinger-picture", Talk presented at the 4th symposium on current trends in physics, Bhubaneswar, India Feb. 24-28 (1986).

[7] F. Cooper, So-Young Pi, and P. N. Stancioff, Phys. Rev. D 34, 3831, (1986).

[8] H. R. Lewis Jr., Phys. Rev. Lett. 27, 510 (1967); H. R. Lewis Jr. and W. B. Riesenfeld, J. Math. Phys. 10, 1458 (1969); S. P. Kim, Class. Quantum, Grav. 13, 1377 (1996); Phys. Rev. D 55, 7511 (1997); D. Bak, S. P. Kim, S. K. Kim, K-S. Soh, and J. H. Yee, J. Korean. Phys. Soc. 37, 168 (2000).

[9] H.-C. Kim, M.-H. Lee, J.-Y. Ji, and J. K. Kim, Phys. Rev. A 53, 3767 (1996).

[10] S. P. Kim and C. H. Lee, Phys. Rev. D 62, 125020 (2000).

[11] Hyeong-Chan Kim and Jae Hyung Yee, Phys. Rev. A 66, 032117 (2002).

[12] P. M. Morse and H. Feshbach, "Methods of Theoretical Physics", New York, (1953). 